\title{
MEMBERS OF THE COUNCIL
}

OfFICERS

Virgil SNyder, President.

O. D. KeLlogG

H. L. RiETZ

J. W. YOUNG

Vice-Presidents.

R. G. D. Richardson, Secretary.

ARNOLD DRESDEN $\}$ Associate Secretaries.

W. B. FITE, Treasurer.

R. C. ARChibald, Librarian.

E. R. HEDRICK

D. R. Curtiss

W. R. LONGLEY

DUNHAM JACKSON

EDWARD KaSNER

H. H. Mrtchell

E. W. Chittenden

A. B. Coble

G. C. Evans

Bulletin Editorial Committee.

Transactions Editorial Committee.

Representatives on Board of Editors of American Journal of Mathematics.

EX-Presidents

G. A. Bliss,

Oswald Veblen,

G. D. BIRKHOFF.

\section{Elected Members}

To serve until December, 1928

TOMLINSON FORT, Olive C. Hazlett,

J. F. RITT,

NORBERT WIENER.

E. B. Stouffer,

To serve until December, 1929

A. A. Bennetr,

J. W. GLOVER,

JAMES Pierpont,

G. E. WAHLIN.

H. S. VANDIVER,

To serve until December, 1930

W. B. Carver,

LOUIS INGOLD,

W. C. Graustein,

W. L. G. Williams.

F. D. MuRnaGhan,

\section{BOARD OF TRUSTEES}

1928

W. B. Fite,

R. G. D. RICHARDSON,

ROBERT HENDERSON, VIRGIL SNYDER,

Óswald Veblen. 